\title{
Distinct Conformers of Assembled Tau in Alzheimer's and Pick's Diseases
}

\author{
Michel Goedert, ${ }^{1}$ Benjamin Falcon, ${ }^{1}$ Wenjuan Zhang, ${ }^{1}$ Bernardino Ghetti, ${ }^{2}$ \\ AND SJORS H.W. Scheres ${ }^{1}$ \\ ${ }^{1}$ MRC Laboratory of Molecular Biology, Cambridge CB2 OQH, United Kingdom \\ ${ }^{2}$ Department of Pathology and Laboratory Medicine, Indiana University School of Medicine, \\ Indianapolis, Indiana 46202, USA \\ Correspondence: mg@mrc-lmb.cam.ac.uk
}

\begin{abstract}
Tau filaments with distinct morphologies and/or isoform compositions underlie a large number of human neurodegenerative diseases. In conjunction with experimental studies, this has led to the suggestion that conformers of aggregated tau exist. Electron cryo-microscopy can be used to determine high-resolution structures of amyloid filaments from human brain. Paired helical and straight tau filaments of Alzheimer's disease (AD) are ultrastructural polymorphs. Each filament core is composed of two identical protofilaments extending from G273/304-E380 (in the numbering of the 441-amino acid isoform of human tau), which adopt a combined cross- $\beta / \beta$-helix structure. They comprise the ends of the first or second microtubule-binding repeat (R1 or R2), the whole of R3 and R4, and 12 amino acids after R4. In contrast, the core of the narrow filaments of Pick's disease (PiD) consists of a single protofilament extending from K254-F378 of $3 \mathrm{R}$ tau, which adopts a cross- $\beta$ structure. It comprises the last 21 amino acids of R1, all of R3 and R4, and 10 amino acids after R4. Wide tau filaments of PiD, which are in the minority, consist of two narrow filaments packed against each other. The tau filament folds of AD and PiD appear to be conserved between different cases of disease. These findings show that filamentous tau adopts one fold in AD and a different fold in PiD, establishing the existence of distinct conformers.
\end{abstract}

The ordered assembly of fewer than 10 different proteins into filamentous assemblies defines the majority of cases of age-related neurodegenerative diseases, including Alzheimer's and Parkinson's. Most cases of disease are sporadic, but a small percentage is inherited in a dominant manner. Huntington's disease is an exception, because it is always inherited. Work performed over the past 35 years established a causative role for filament formation in inherited forms of disease. By extrapolation, it appears likely that inclusion formation is central to neurodegeneration in all sporadic cases of disease. Tauopathies, which are characterized by the assembly of tau protein, are the most common proteinopathies of the human nervous system. They include cases of Alzheimer's disease (AD), Pick's disease (PiD), chronic traumatic encephalopathy (CTE), tangle-only dementia, progressive supranuclear palsy (PSP), corticobasal degeneration (CBD), argyrophilic grain disease (AGD), and several rarer diseases.

\section{TAU PROTEIN}

Tau belongs to the family of intrinsically disordered proteins, which have many interaction partners and are often implicated in neurodegenerative diseases (Goedert et al. 2017). Tau can be divided into an amino-terminal domain, a proline-rich region, the repeat domain, and a carboxy-terminal domain. The amino-terminal domain projects away from the microtubule surface and is believed to interact with components of the neuronal plasma membrane. An interaction between exon 1 and annexins may help to explain the axonal localization of tau (GauthierKemper et al. 2018). Exon 1 contains a primate-specific sequence between residues 18 and 28 of human tau that has been proposed to mediate interactions with neuronal proteins (Stefanoska et al. 2018). The PXXP motifs in the proline-rich region are recognized by SH3 domain-containing proteins of the Src family of nonreceptor tyrosine kinases, such as Fyn (Lee et al. 1998).

The repeat domain and some adjacent sequences mediate interactions between tau and microtubules. Electron cryo-microscopy (cryo-EM) has shown that each tau repeat binds to the outer microtubule surface and adopts an extended structure along protofilaments, interacting with $\alpha$ - and $\beta$-tubulin (Al-Bassam et al. 2002; Kellogg et al. 2018). Single-molecule tracking revealed a kiss-and-hop mechanism, with a dwell time of tau on individual microtubules of $\sim 40 \mathrm{msec}$ (Janning et al. 2014; Niewidok et al. 2016). Isoform differences do not influence this interaction. Despite these rapid dynamics, tau promotes microtubule assembly. It remains to be seen if microtubules are also stabilized. Individual axonal microtubules have stable and labile domains. Tau is most abundant in the labile domain, which has led to the suggestion that it may not stabilize microtubules but enable them to have long labile domains (Black et al. 1996; Qiang et al. 2018). Less is known about the function of the carboxy-terminal region, which may inhibit assembly into filaments. Although it

(C) 2018 Goedert et al. This article is distributed under the terms of the Creative Commons Attribution-NonCommercial License, which permits reuse and redistribution, except for commercial purposes, provided that the original author and source are credited. 
lacks a typical low-complexity domain, full-length tau has been shown to undergo liquid-liquid phase separation, which has been reported to initiate aggregation, at least in vitro (Zhang et al. 2017b; Wegmann et al. 2018). Tau is subject to a large number of posttranslational modifications, including phosphorylation, acetylation, methylation, glycation, isomerization, O-GlcNAcylation, nitration, sumoylation, ubiquitination, and truncation (Goedert et al. 2017).

\section{TAU ISOFORMS}

Six tau isoforms ranging from 352 to 441 amino acids in length are expressed in adult human brain from a single $M A P T$ gene (Fig. 1A; Goedert et al. 1989). They differ by the presence or absence of inserts of 29 and 58 amino acids (encoded by exons 2 and 3, with exon 3 being only transcribed in conjunction with exon 2) in the amino-terminal half, and the inclusion, or not, of the 31-amino acid microtubule-binding repeat, encoded by exon 10 , in the carboxy-terminal half. Inclusion of exon 10 results in the production of three isoforms with four repeats (4R) and its exclusion in a further three isoforms with three repeats (3R). The repeats comprise residues 244-368, in the numbering of the 441-amino acid isoform. In adult human brain, similar levels of $3 \mathrm{R}$ and $4 \mathrm{R}$ tau are expressed; the finding that a correct isoform ratio is essential for preventing neurodegeneration came as a surprise. $2 \mathrm{~N}$ isoforms are underrepresented in comparison with isoforms that include exon 2 or exclude both exons 2 and $3 ; 2 \mathrm{~N}, 1 \mathrm{~N}$, and $0 \mathrm{~N}$ tau isoforms make up $9 \%, 54 \%$, and $37 \%$, respectively. Big tau, which carries an additional large exon in the amino-terminal half, is only expressed in the peripheral nervous system (Couchie et al. 1992; Goedert et al. 1992).

Tau isoform expression is not conserved between species. Thus, in adult mouse brain, $4 \mathrm{R}$ tau isoforms are almost exclusively present, whereas adult chicken brain expresses 3R, 4R, and 5R tau isoforms. However, the presence of one hyperphosphorylated $3 \mathrm{R}$ tau isoform lacking amino-terminal repeats is characteristic of developing vertebrates. In mice, the switch from $3 \mathrm{R}$ to $4 \mathrm{R}$ tau occurs between postnatal days 9 and 18, with tau phos-

A

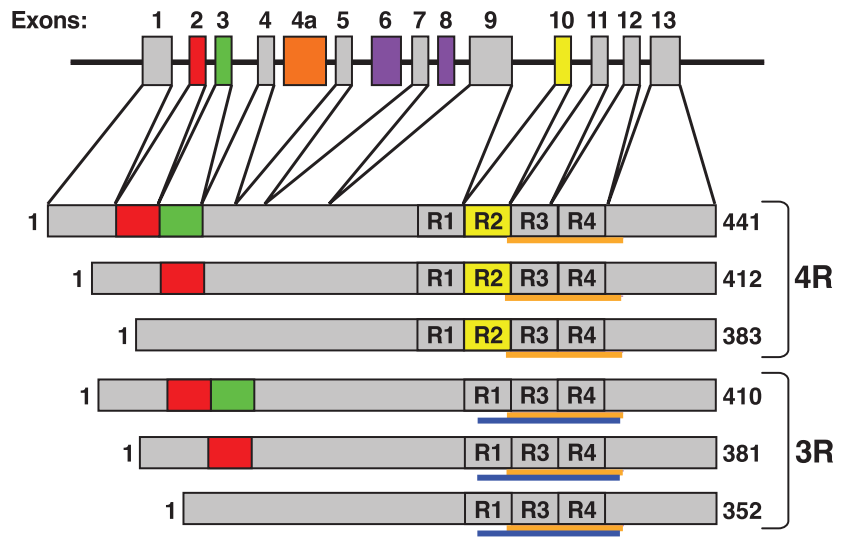

B

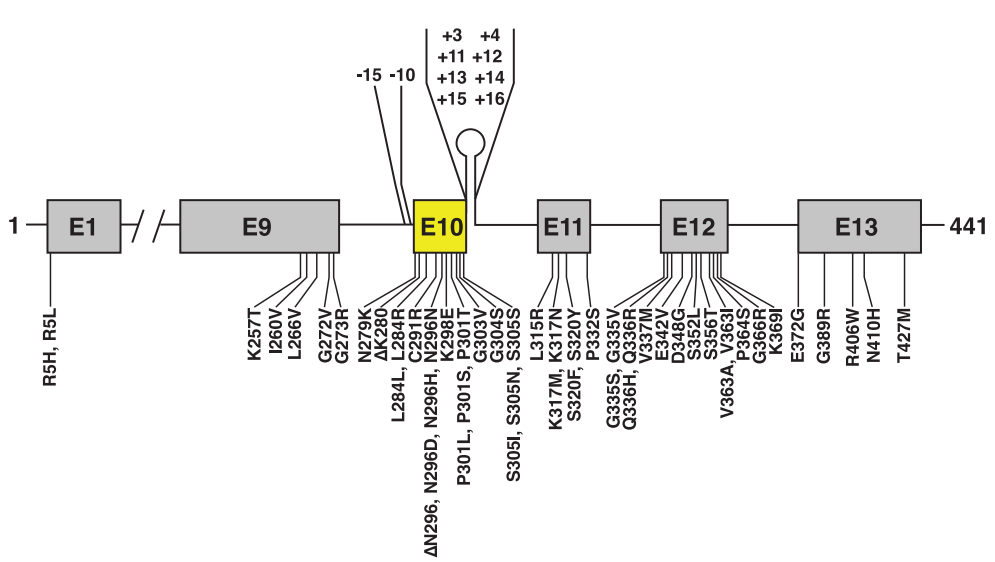

Figure 1. Human brain tau isoforms and disease-causing MAPT mutations. (A) MAPTand the six tau isoforms expressed in adult human brain. MAPT consists of 14 exons (E). Alternative mRNA splicing of E2 (red), E3 (green), and E10 (yellow) gives rise to six tau isoforms (352-441 amino acids). The constitutively spliced exons (E1, E4, E5, E7, E9, E11, E12, and E13) are shown in gray. E6 and E8 (violet) are not transcribed in human brain. E4a (orange) is only expressed in the peripheral nervous system. The repeats (R1-R4) are shown, with three isoforms having four repeats (4R) and three isoforms with three repeats (3R). The core sequences of tau filaments from Alzheimer's disease (G273/305-E380) determined by cryo-EM are underlined (in orange); the core sequences of tau filaments from Pick's disease (K254-F378 of 3R tau) are also underlined (in blue). (B) Mutations in MAPT in FTDP-17T. Fifty coding region mutations and 10 intronic mutations flanking E10 are shown. 
phorylation decreasing over time (Tuerde et al. 2018). However, isoform switching and phosphorylation are regulated differently. Adult $4 \mathrm{R}$ tau isoforms are better at promoting microtubule assembly and binding to microtubules than the 3R tau isoform expressed during development (Goedert and Jakes 1990). This is consistent with the need for a more dynamic cytoskeleton during the development of nerve cells.

\section{TAU ASSEMBLIES}

Full-length tau assembles into filaments; the repeats and some adjoining sequences form the filament core, with the amino-terminal half and the carboxyl terminus giving rise to the fuzzy coat (Goedert et al. 1988; Wischik et al. 1988a,b). Tau filaments from human brain and those assembled in vitro from overexpressed protein have a cross- $\beta$ structure characteristic of amyloid (Berriman et al. 2003). Because the region that binds to microtubules also forms the core of tau filaments, physiological function and pathological assembly may be mutually exclusive.

Phosphorylation negatively regulates the ability of tau to interact with microtubules and filamentous tau is abnormally hyperphosphorylated (Iqbal et al. 2016). However, it remains to be proved that phosphorylation is the trigger for aggregation in human diseases. Alternatively, a change in conformation as part of aggregation may lead to tau hyperphosphorylation. Because tau is hydrophilic, it is not surprising that the unmodified full-length protein requires cofactors, such as heparin, to assemble into filaments (Goedert et al. 1996; Pérez et al. 1996). Cofactors other than heparin and/or posttranslational modifications may cause the assembly of tau in human brain.

Besides phosphorylation, other modifications may also be involved. Early studies on tau acetylation reported that it can promote both phosphorylation and aggregation (Min et al. 2010; Cohen et al. 2011). However, subsequent work suggested an inverse correlation between tau acetylation and phosphorylation, with acetylation inhibiting tau assembly (Cook et al. 2014; Carlomagno et al. 2017). These discrepancies may have resulted from the use of enzymes that acetylated different residues. The site-specific acetylation of K280 has been shown to enhance tau aggregation while reducing microtubule assembly (Haj-Yahya and Lashuel 2018). Unlike phosphorylation, acetylation occurs on lysine residues.

In $\mathrm{AD}, \mathrm{CTE}$, tangle-only dementia, and many other tauopathies, all six tau isoforms are present in disease filaments (Table 1). The Pick bodies of PiD are only made of 3R tau. In PSP, CBD, AGD, and several other diseases, isoforms with $4 \mathrm{R}$ tau are found in the filaments. The morphologies of tau filaments vary in the different diseases, even when they are made of the same isoforms.

\section{GENETICS OF MAPT}

The relevance of tau inclusion formation for neurodegeneration became clear in June 1998, when dominantly inherited mutations in MAPT were shown to cause a form
Table 1. Neurodegenerative diseases with abundant Tau inclusions

$3 \mathrm{R}+4 \mathrm{R}$ tauopathies

Alzheimer's disease

Amyotrophic lateral sclerosis/parkinsonism-dementia complex (Guam and Kii peninsula)

Anti-IgLON5-related tauopathy

Chronic traumatic encephalopathy

Diffuse neurofibrillary tangles with calcification

Down's syndrome

Familial British dementia

Familial Danish dementia

Gerstmann-Sträussler-Scheinker disease

Niemann-Pick disease, type C

Nodding syndrome

Non-Guamanian motor neuron disease with neurofibrillary tangles

Postencephalitic parkinsonism

Primary age-related tauopathy

Progressive ataxia and palatal tremor

Tangle-only dementia

Familial frontotemporal dementia and parkinsonism (some $M A P T$ mutations, such as V337M and R406W)

3R tauopathies

Pick's disease

Familial frontotemporal dementia and parkinsonism (some $M A P T$ mutations, such as G272V and Q336R)

4R tauopathies

Age-related tau astrogliopathy

Argyrophilic grain disease

Corticobasal degeneration

Guadeloupean parkinsonism

Globular glial tauopathy

Hippocampal tauopathy

Huntington's disease

Progressive supranuclear palsy

SLC9a-related parkinsonism

Familial frontotemporal dementia and parkinsonism (some MAPT mutations, such as P301L and P301S, all known intronic mutations, and many coding region mutations in exon 10)

of frontotemporal dementia that can be associated with parkinsonism (FTDP-17T, also known as familial FTLD-tau) (Hutton et al. 1998; Poorkaj et al. 1998; Spillantini et al. 1998). In FTDP-17T, abundant filamentous tau inclusions are present in either nerve cells or in both nerve cells and glial cells. $A \beta$ deposits, a defining feature of AD, are not characteristic of FTDP-17T. This work established that a pathological pathway leading from monomeric to assembled tau is sufficient for causing neurodegeneration and dementia.

Sixty mutations in MAPT have been identified in FTDP-17T (Fig. 1B). Filaments are composed of either $3 \mathrm{R}$ or $4 \mathrm{R}$ tau or of both $3 \mathrm{R}$ and $4 \mathrm{R}$ tau. MAPT mutations account for $\sim 5 \%$ of cases of FTLD and are concentrated in exons 9-12 (encoding R1-R4) and the introns flanking exon 10 . They can be divided into those with a primary effect at the protein level and those affecting the alternative splicing of tau pre-mRNA. There is no obvious correlation between known mutations and posttranslational modifications of tau.

The architecture of MAPT on chromosome 17q21.31 is characterized by two haplotypes as the result of a $900-\mathrm{kb}$ inversion (H1) or noninversion (H2) polymorphism (Stefansson et al. 2005). Inheritance of the H1 haplotype of 
$M A P T$ is a risk factor for $\mathrm{PSP}, \mathrm{CBD}, \mathrm{PD}$, and amyotrophic lateral sclerosis (ALS), but not for PiD (Conrad et al. 1997; Baker et al. 1999; Pastor et al. 2000; Houlden et al. 2001; Morris et al. 2002; Zhang et al. 2017a). The $\mathrm{H} 2$ haplotype is associated with increased expression of exon 3 of $M A P T$ in gray matter, suggesting that inclusion of exon 3 may protect against PSP, CBD, PD, and ALS (Caffrey et al. 2008). In experimental studies, exon 3-containing tau isoforms (those with both amino-terminal inserts) have been found to aggregate less than those lacking this exon (Zhong et al. 2012).

Heterozygous microdeletions of chromosome 17q21.31 give rise to a multisystem disorder with intellectual disability, hypotonia, and distinct facial features (17q21.31 microdeletion syndrome or Koolen-de Vries syndrome) (Koolen et al. 2006; Sharp et al. 2006; Shaw-Smith et al. 2006). In addition to $M A P T$, three protein-coding genes (CRHR1, SPPL2C, and KANSL1) and two putative genes (MGC57346 and CRHR1-ITI) are found in this region. Deletions arise on the H2 haplotype through low-copy, repeat-mediated, nonallelic homologous recombination. The 17q21.31 microdeletion syndrome is caused by haploinsufficiency of KANSL1, which encodes a chromatin modifier that influences gene expression through the acetylation of lysine 16 of histone H4 (Koolen et al. 2012; Zollino et al. 2012). A 50\% reduction in tau levels does therefore not appear to have a detrimental effect on development of the human brain.

Disease-causing mutations in MAPT have made it possible to produce transgenic rodent lines that form tau filaments and show neurodegeneration (Goedert et al. 2017). Aggregation of tau correlates with neurodegeneration. Reducing aggregation and increasing degradation of aggregates are therefore therapeutic objectives. It has been reported that the removal of senescent brain cells leads to a reduction in both tau aggregates and neurodegeneration in transgenic mice (Bussian et al. 2018).

Transgenic mouse lines were also essential for the in vivo discovery of the prion-like properties of assembled tau. Aggregation of hyperphosphorylated tau was induced following intracerebral injection of tau seeds from mice transgenic for human mutant 0N4R P301S tau into transgenic mice expressing wild-type nonaggregated 2N4R tau and, to a lesser extent, following intracerebral injection into wild-type mice (Clavaguera et al. 2009). Tauopathy then spread to connected brain regions, indicative of seed endocytosis, seeded aggregation, intracellular transport, and release of tau seeds. This work was complemented by studies in cells, which showed that short tau filaments had the greatest seeding activity (Jackson et al. 2016). These findings may be mechanistically related to the observation that in the process leading to $\mathrm{AD}$, tau inclusions first appear in locus coeruleus and entorhinal cortex, followed by the hippocampal formation and large parts of the neocortex (Braak and Braak 1991).

Distinct conformers of assembled tau appear to exist, reminiscent of prion strains. They may explain the variety of human tauopathies. Inclusions formed and spread of pathology occurred after intracerebral injection of brain homogenates from cases of $\mathrm{AD}$, tangle-only dementia, PSP, CBD, and AGD into a mouse line transgenic for wild-type 4R tau and, to a lesser extent, following intracerebral injection into nontransgenic mice (Clavaguera et al. 2013). PiD, the filamentous inclusions of which consist of only $3 \mathrm{R}$ tau, was an exception. Inclusions formed at the injection sites, but spreading was not observed. However, PiD is rarely a pure $3 \mathrm{R}$ tauopathy, because it is often associated with a low level of AD-type pathology. We therefore cannot exclude that the aggregation-inducing activity in the PiD homogenate may have been due to the presence of a small amount of aggregated 4R tau. The sequence requirements for seeded tau aggregation in vivo remain to be defined. Tau assemblies reminiscent of those in the corresponding human diseases were observed following the injection of brain homogenates from patients with PSP, CBD, and AGD, which are 4R tauopathies. Although these findings are consistent with the existence of distinct tau aggregate conformers, the definition of an aggregate conformer ought to be structural.

\section{HIGH-RESOLUTION STRUCTURES OF TAU FILAMENTS FROM ALZHEIMER'S DISEASE}

By cryo-EM, high-resolution structures of tau filaments were obtained from the frontal cortex of four individuals with $\mathrm{AD}$, three sporadic and one inherited (mutation V717F in $A P P$ ) (Fig. 2; Fitzpatrick et al. 2017; Falcon et al. 2018a). The cores of tau filaments are made of two protofilaments consisting of residues G273/304-E380, which adopt a combined cross- $\beta / \beta$-helix structure (Fig. 3 ). Mouse tau is identical in sequence to human tau in this region. The amino-terminal part of the cross- $\beta$ structure includes the hexapeptide ${ }^{306}$ VQIVYK $^{311}$ (PHF6), which is essential for the oligomerization of recombinant tau and its assembly into filaments (Fig. 3; von Bergen et al. 2000). It packs against ${ }^{373} \mathrm{THKLTF}^{378}$, in agreement with the predicted heterozipper interaction between ${ }^{306} \mathrm{VQIVYK}^{311}$ and ${ }^{375}$ KLTFR $^{379}$ (Moore et al. 2011). Constructs K18 and K19 end at E372 (Gustke et al. 1994); they can therefore not give rise to the human brain tau folds determined thus far.

Each protofilament contains eight $\beta$-strands, five of which give rise to two regions of antiparallel $\beta$-sheets, with the other three forming a $\beta$-helix (Fig. 4). The carboxy-terminal residues of R1 and R2 form part of the first $\beta$-strand. R3 contributes three and R4 four $\beta$-strands, with the final $\beta$-strand being formed by the 12 amino acids after $\mathrm{R} 4 ; \beta 1$ and $\beta 2$ pack against $\beta 8, \beta 3$ packs against $\beta 7$, with $\beta 4, \beta 5$, and $\beta 6$ giving rise to the $C$-shaped $\beta$-helix.

Paired helical filaments (PHFs) and straight filaments (SFs) are made of identical protofilaments, but differ in interprotofilament packing, showing that they are ultrastructural polymorphs. PHF protofilaments are arranged base-to-base and SF protofilaments back-to-base. In PHFs, protofilaments are stabilized by backbone hydrogen bonds between their ${ }^{332} \mathrm{PGGGQ}^{336}$ sequences. Moreover, the side chains of K331 from one protofilament project toward the side chains of Q336 and E338 of the 

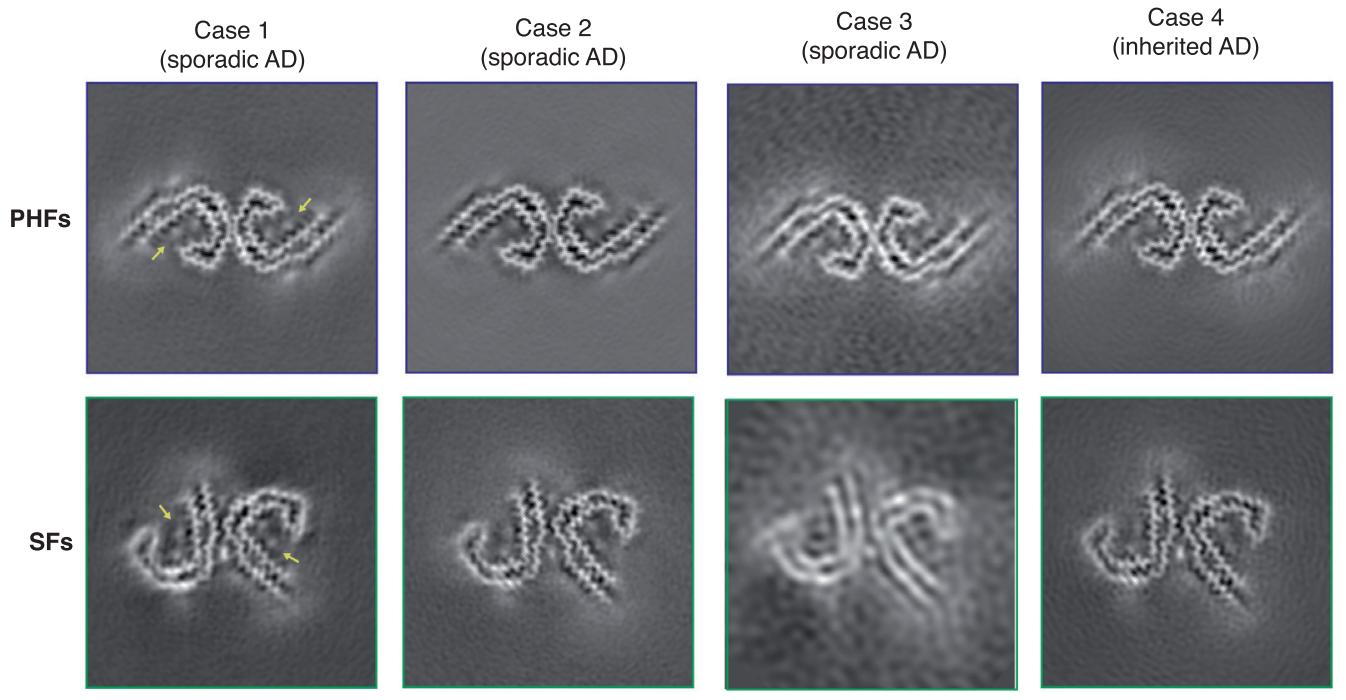

Figure 2. Cryo-EM structures of paired helical filaments (PHFs) and straight filaments (SFs) from the frontal cortex of four Alzheimer's disease (AD) cases. All structures show identical pairs of C-shaped protofilaments and the same interprotofilament packing in PHFs and SFs. Cases 1, 2, and 3 had sporadic AD, whereas case 4 had inherited AD (mutation V717F in the amyloid precursor protein gene). All cases had a majority of PHFs and a minority of SFs. Yellow arrows point to the extra densities, which were present in PHFs and SFs from all four cases, bordering the solvent-exposed side chains of R349 and K375 and of H362 and K369.

other protofilament, suggesting additional interactions that stabilize the protofilament interface. Furthermore, in the protofilament interface of the PHF, extra densities between the side chains of K331 of one protofilament and the backbone of V337 of the other have been observed. They may correspond to a solvent molecule or a posttranslational modification of K331, such as monomethylation (Falcon et al. 2018a).

In $\mathrm{SFs}$, the protofilaments pack asymmetrically. Their backbones are nearest each other between residues ${ }^{321} \mathrm{KCGS}^{324}$ of the first and ${ }^{313}$ VDLSK $^{317}$ of the second protofilament. The interprotofilament packing appears to be stabilized through the region of additional density that interacts with the side chains of K317, T319, and K321 of both protofilaments. This density may correspond to resi- dues ${ }^{7} \mathrm{EFE}^{9}$, which constitute the amino-terminal region of the discontinuous epitope of the conformational antitau antibodies ALZ-50 and MC-1 (the carboxy-terminal epitope is ${ }^{313}$ VDLSKVTSKC ${ }^{322}$ ) (Jicha et al. 1997). A similar density also interacts with K317, T319, and K321 in PHFs, where it does not contribute to the protofilament interface.

\section{HIGH-RESOLUTION STRUCTURES OF TAU FILAMENTS FROM PICK'S DISEASE}

By cryo-EM, high-resolution structures of tau filaments were determined from the frontotemporal cortex of an individual with PiD (Falcon et al. 2018b). Two types of filaments could be distinguished: a majority of narrow
PHFs

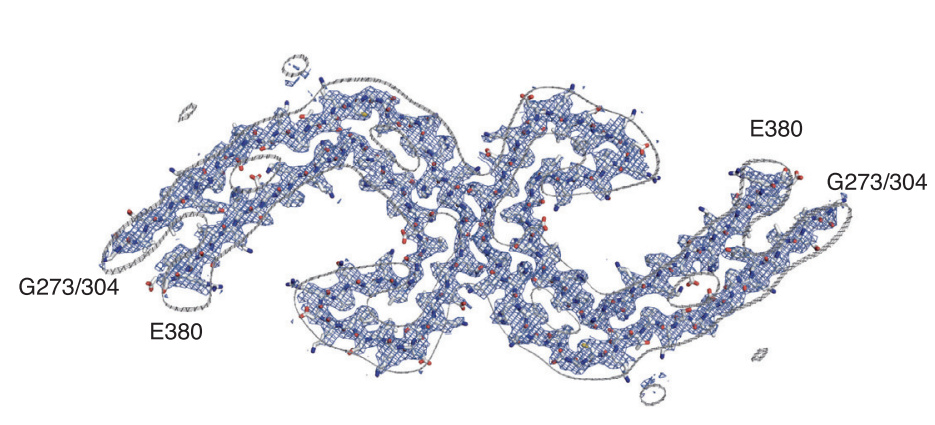

SFs

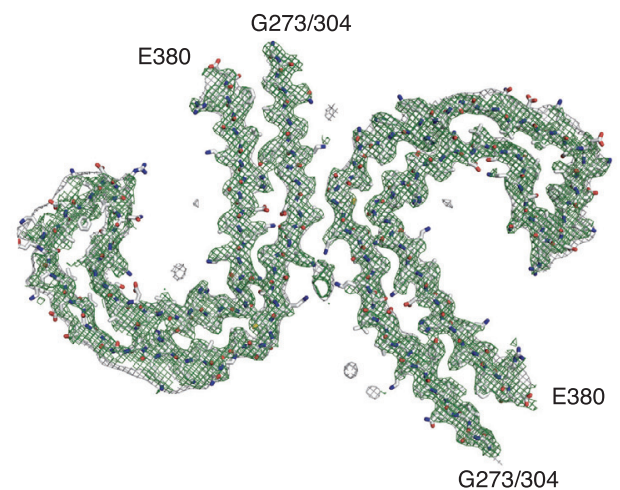

Figure 3. Cryo-EM densities and atomic models of paired helical filaments (PHFs) and straight filaments (SFs) from the frontal cortex of an individual with Alzheimer's disease. PHFs and SFs were resolved to 3.2 and $3.3 \AA$, respectively. Sharpened, high-resolution maps are shown in blue (PHFs) and green (SFs). Unsharpened $4.5 \AA$ low-pass filtered densities are shown in gray. The models comprise G273E380 of 3R tau and G304-E380 of 4R tau. The protofilaments of PHFs and SFs are identical but differ in interprotofilament packing (ultrastructural polymorphs). 


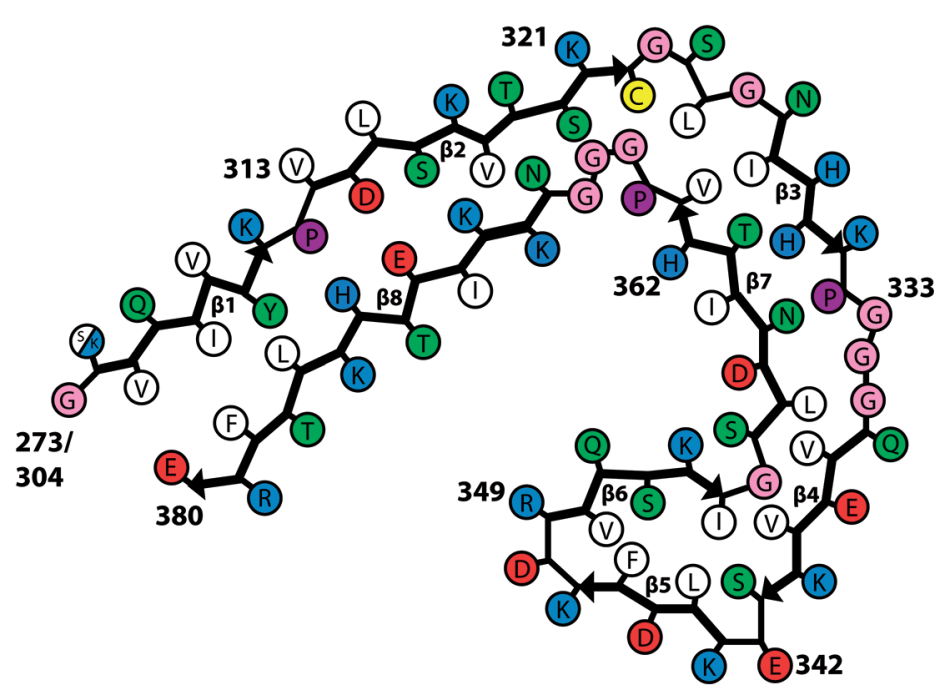

Figure 4. Schematic view of the tau protofilament core of Alzheimer's disease. The observed eight $\beta$ strand regions $(\beta 1-\beta 8)$ are shown as arrows. Each filament consists of two protofilaments.
Pick filaments (NPFs) and a minority of wide Pick filaments (WPFs) (Fig. 5). The core of NPFs is made of a single protofilament that consists of residues K254-F378 of $3 \mathrm{R}$ tau, which adopt an elongated cross- $\beta$ structure. Mouse tau is identical to human tau in this region, with the exception of residue 257 ( $\mathrm{K}$ in human, $\mathrm{R}$ in mouse tau). WPFs are formed by the association of two NPF protofilaments at their distal tips, where they form tight contacts through van der Waals interactions. Each protofilament comprises nine $\beta$-strands, which are arranged into four cross- $\beta$ packing stacks and are connected by turns and arcs (Fig. 6). R1 provides two $\beta$-strands and
R3 and R4 three $\beta$-strands each. These stacks pack together in a hairpin-like fashion: $\beta 1$ against $\beta 8, \beta 2$ against $\beta 7$, $\beta 3$ against $\beta 6$, and $\beta 4$ against $\beta 5$. The final strand, $\beta 9$, is formed from the 10 amino acids after R4 and packs against the opposite side of $\beta 8$.

Three regions of less well-resolved density bordering the solvent-exposed faces of $\beta 4, \beta 5$, and $\beta 9$ are apparent in both NPFs and WPFs. They may represent less ordered, heterogeneous, and/or transiently occupied structures. The density bordering $\beta 4$ is similarly located, but more extended, than that found to interact with the side chains of $\mathrm{K} 317$, T319, and K321 in AD filaments.
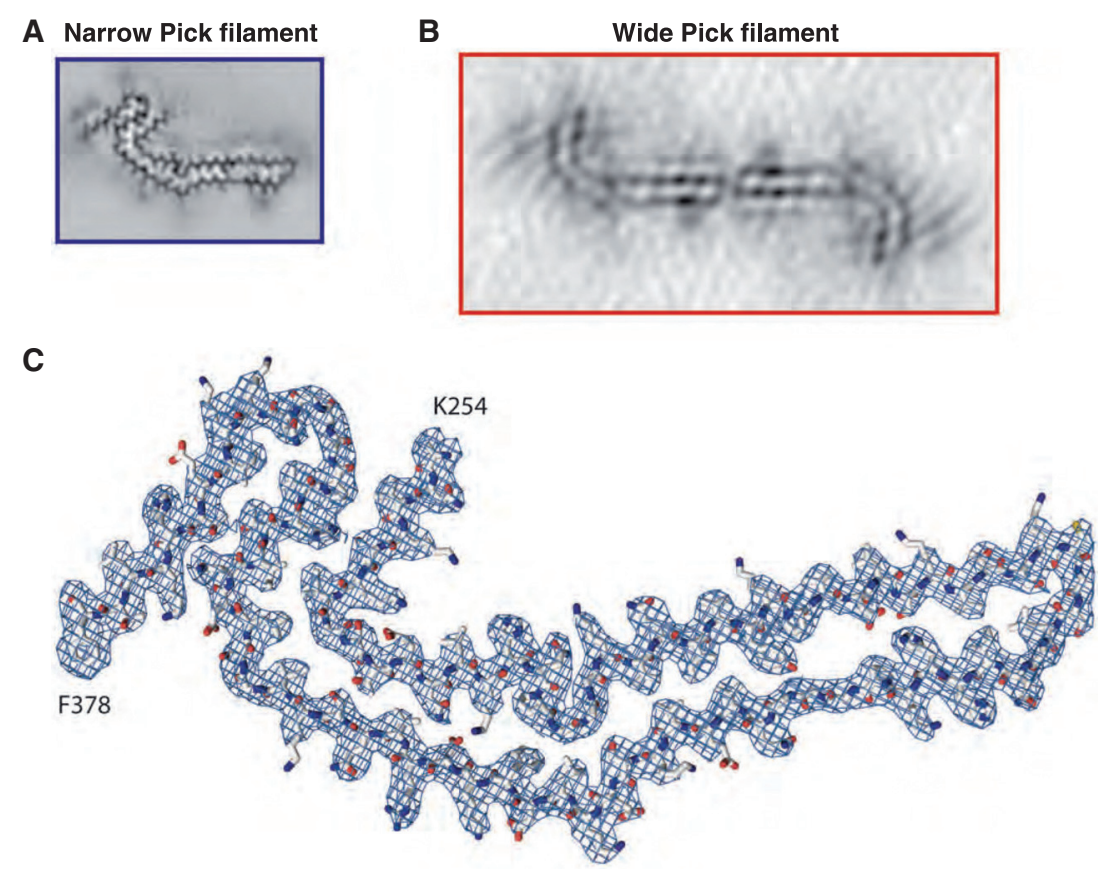

Figure 5. Narrow (93\%) and wide (7\%) tau filaments (NPFs and WPFs) are characteristic of Pick's disease. Unsharpened cryo-EM densities of a NPF $(A)$ and a WPF $(B)$. NPFs were resolved to $3.2 \AA$. (C) Sharpened, high-resolution cryo-EM map of the NPF with the atomic model of the Pick fold overlaid. 


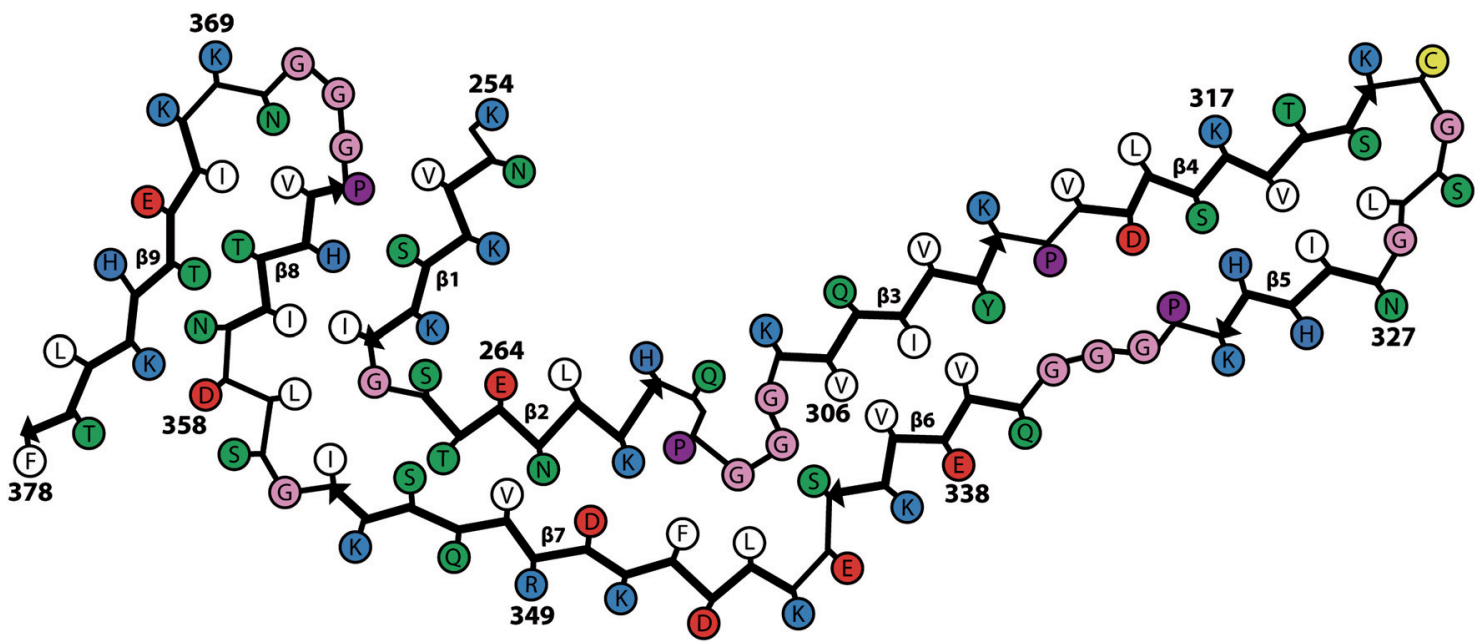

Figure 6. Schematic view of the tau protofilament core of Pick's disease. The observed nine $\beta$-strands ( $\beta 1-\beta 9)$ are shown as arrows. Narrow Pick filaments are made of one protofilament. Wide Pick filaments comprise two narrow filaments that are joined at their distal tips.

It was not previously known why only $3 \mathrm{R}$ tau is present in Pick body filaments and why S262 is not phosphorylated. Our results suggest that despite sequence homology, the structure formed by K254-K274 of R1 is inaccessible to the corresponding residues from R2 (S285-S305). Moreover, because of steric constraints, the filament structure precludes phosphorylation of S262.

\section{CONCLUSION}

Cryo-EM of filaments from human brain has established that distinct conformers of aggregated tau are characteristic of $\mathrm{AD}$ and PiD. Even though both types of filaments share residues G273-F378 of 3R tau, their structures are very different (Fig. 7). Whereas PHFs and SFs of $\mathrm{AD}$ are made of two identical C-shaped protofilaments
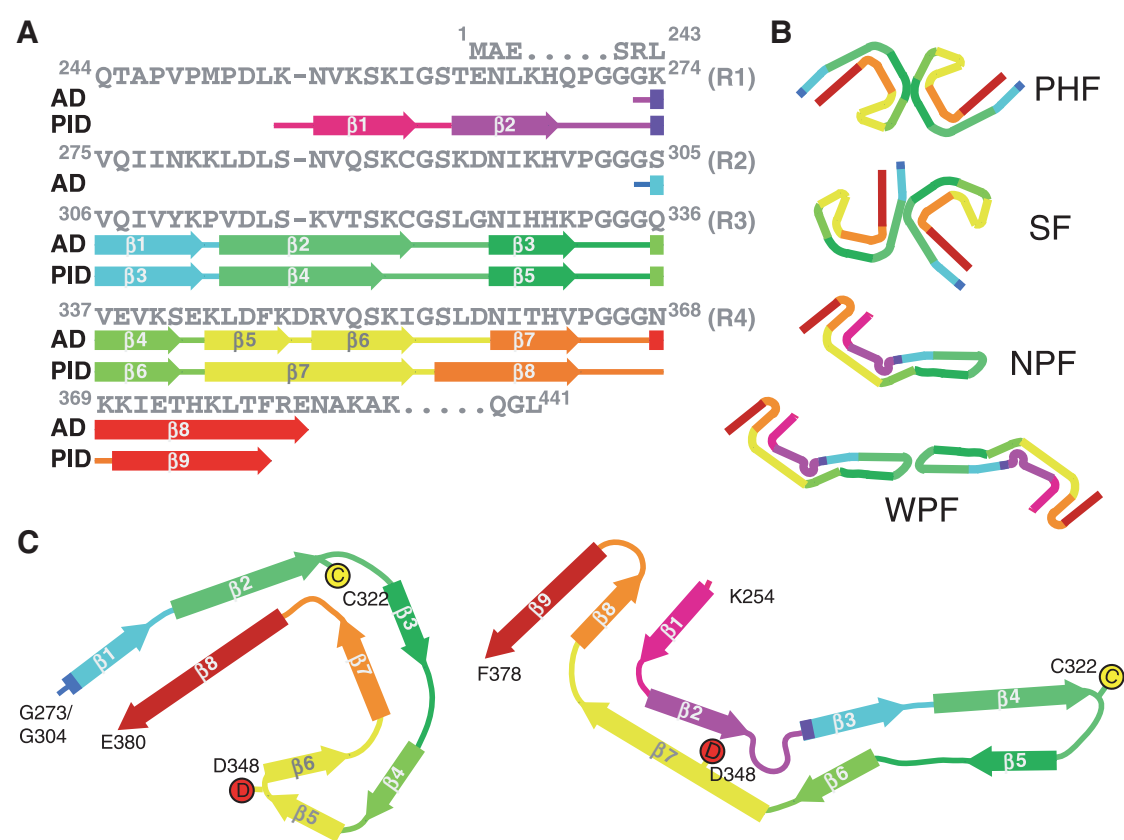

Alzheimer fold

Pick fold

Figure 7. Comparison of Alzheimer and Pick tau filament folds. (A) Microtubule-binding repeats (R1-R4, residues 244-368) and some flanking sequences, with the observed eight $\beta$-strand regions (arrows) in the Alzheimer fold and nine $\beta$-strand regions (arrows) in the Pick fold, as well as corresponding loop regions, colored from violet to red. (B) Schematic representation of the different tau folds. Paired helical (PHF) and straight (SF) tau filaments of Alzheimer's disease consist of two identical protofilaments that differ in interprotofilament packing (ultrastructural polymorphs). More than $90 \%$ of tau filaments of Pick's disease are narrow Pick filaments (NPFs) that consist of a single protofilament. Wide Pick filaments (WPFs) consist of two narrow filaments packed against each other. $(C)$ Schematic of the Alzheimer and Pick folds, depicted as a single rung. C322 (yellow "C") and D348 (red "D") are highlighted. 
that each comprises eight $\beta$-strands and a combined cross$\beta / \beta$-helix structure, NPFs of PiD are made of a single elongated protofilament comprising nine $\beta$-strands and stacks of $\beta$-sheets. WPFs consist of two NPFs joined through their distal tips. Cryo-EM studies of tau filaments from additional sporadic and inherited cases of $\mathrm{AD}$, as well as negative-stain immuno-electron microscopy of tau filaments from multiple cases of $\mathrm{AD}$ and $\mathrm{PiD}$, indicated that the cores of tau filaments from each disease case contain the same sequences. It therefore seems that the structures of tau filaments are distinct between diseases but identical in different individuals with $\mathrm{AD}$ or PiD. It appears likely that additional folds of assembled tau remain to be discovered in other tauopathies.

\section{REFERENCES}

Al-Bassam J, Ozer RS, Safer D, Halpain S, Milligan RA. 2002. MAP2 and tau bind longitudinally along the outer ridges of microtubule protofilaments. J Cell Biol 157: 1187-1196. doi:10.1083/jcb.200201048

Baker M, Litvan I, Houlden H, Adamson J, Dickson D, Perez-Tur J, Hardy J, Lynch T, Bigio E, Hutton M. 1999. Association of an extended haplotype in the tau gene with progressive supranuclear palsy. Hum Mol Genet 8: 711-715. doi:10.1093/hmg/ 8.4.711

Berriman J, Serpell LC, Oberg KS, Fink AL, Goedert M, Crowther RA. 2003. Tau filaments from human brain and from in vitro assembly of recombinant protein show cross- $\beta$ structure. Proc Natl Acad Sci 100: 9034-9038. doi:10.1073/ pnas. 1530287100

Black MM, Slaughter T, Moshiach S, Obrocka M, Fischer I. 1996. Tau is enriched on dynamic microtubules in the distal region of growing axons. J Neurosci 16: 3601-3619. doi:10 .1523/JNEUROSCI.16-11-03601.1996

Braak H, Braak E. 1991. Neuropathological stageing of Alzheimer-related changes. Acta Neuropathol 82: 239-259. doi:10.1007/BF00308809

Bussian TJ, Aziz A, Meyer CF, Swenson BL, Van Deursen JM, Baker DJ. 2018. Clearance of senescent glial cells prevents tau-dependent pathology and cognitive decline. Nature 562: 578-582. doi:10.1038/s41586-018-0543-y

Caffrey TM, Joachim C, Wade-Martins R. 2008. Haplotype-specific expression of the N-terminal exons 2 and 3 at the human MAPT locus. Neurobiol Aging 29: 1923-1929. doi:10.1016/j .neurobiolaging.2007.05.002

Carlomagno Y, Chung DC, Yue M, Castanedes-Casey M, Madden BJ, Dunmore J, Tong J, DeTure M, Dickson DW, Petrucelli L, et al. 2017. An acetylation-phosphorylation switch that regulates tau aggregation propensity and function. $J$ Biol Chem 292: 15277-15286. doi:10.1074/jbc.M117 .794602

Clavaguera F, Bolmont T, Crowther RA, Abramowski D, Frank S, Probst A, Fraser G, Stalder AK, Beibel M, Staufenbiel M, et al. 2009. Transmission and spreading of tauopathy in transgenic mouse brain. Nat Cell Biol 11: 909-913. doi:10.1038/ ncb1901

Clavaguera F, Akatsu H, Fraser G, Crowther RA, Frank S, Hench J, Probst A, Winkler DT, Reichwald J, Staufenbiel M, et al. 2013. Brain homogenates from human tauopathies induce tau inclusions in mouse brain. Proc Natl Acad Sci 110: 95359540. doi: $10.1073 /$ pnas. 1301175110

Cohen TJ, Guo JL, Hurtado DE, Kwong LK, Mills IP, Trojanowski JQ, Lee VMY. 2011. The acetylation of tau inhibits its function and promotes pathological tau aggregation. Nat Commun 2: 252. doi:10.1038/ncomms 1255

Conrad C, Andreadis A, Trojanowski JQ, Dickson DW, Kang D, Chen X, Wiederholt W, Hansen L, Masliah E, Thal LJ, et al.
1997. Genetic evidence for the involvement of $\tau$ in progressive supranuclear palsy. Ann Neurol 41: 277-281. doi:10.1002/ana .410410222

Cook C, Carlomagno Y, Gendron TF, Dunmore J, Scheffel K, Stetler C, Davis M, Dickson D, Jarpe M, DeTure M, et al. 2014. Acetylation of the KXGS motifs in tau is a critical determinant in modulation of tau aggregation and clearance. Hum Mol Genet 23: 104-116. doi:10.1093/hmg/ ddt402

Couchie D, Mavilia C, Georgieff IS, Liem RK, Shelanski ML, Nunez J. 1992. Primary structure of high molecular weight tau present in the peripheral nervous system. Proc Natl Acad Sci 89: 4378-4381. doi:10.1073/pnas.89.10.4378

Falcon B, Zhang W, Schweighauser M, Murzin AG, Vidal R, Garringer HJ, Ghetti B, Scheres SHW, Goedert M. 2018a. Tau filaments from multiple cases of sporadic and inherited Alzheimer's disease adopt a common fold. Acta Neuropathol 136: 699-708. doi:10.1007/s00401-018-1914-Z

Falcon B, Zhang W, Murzin AG, Murshudov G, Garringer HJ, Vidal R, Crowther RA, Ghetti B, Scheres SHW, Goedert M. 2018b. Structures of filaments from Pick's disease reveal a novel tau protein fold. Nature 561: 137-140. doi:10.1038/ s41586-018-0454-y

Fitzpatrick AWP, Falcon B, He S, Murzin AG, Murshudov G, Garringer HJ, Crowther RA, Ghetti B, Goedert M, Scheres SHW. 2017. Cryo-EM structures of tau filaments from Alzheimer's disease. Nature 547: 185-190. doi:10.1038/ nature 23002

Gauthier-Kemper A, Suárez Alonso M, Sündermann F, Niewidok B, Fernandez MP, Bakota L, Heinisch JJ, Brandt R. 2018. Annexins A2 and A6 interact with the extreme Nterminus of tau and thereby contribute to tau's axonal localization. J Biol Chem 293: 8065-8076. doi:10.1074/jbc.RA117 .000490

Goedert M, Jakes R. 1990. Expression of separate isoforms of human tau protein: correlation with the tau pattern in brain and effects on tubulin polymerization. EMBO $J$ 9: 4225-4230. doi:10.1002/j.1460-2075.1990.tb07870.x

Goedert M, Wischik CM, Crowther RA, Walker JE, Klug A. 1988. Cloning and sequencing of the cDNA encoding a core protein of the paired helical filament of Alzheimer disease: identification as the microtubule-associated protein tau. Proc Natl Acad Sci 85: 4051-4055. doi:10.1073/pnas.85.11 .4051

Goedert M, Spillantini MG, Jakes R, Rutherford D, Crowther RA. 1989. Multiple isoforms of human microtubule-associated protein tau: sequences and localization in neurofibrillary tangles of Alzheimer's disease. Neuron 3: 519-526. doi:10 .1016/0896-6273(89)90210-9

Goedert M, Spillantini MG, Crowther RA. 1992. Cloning of a big tau microtubule-associated protein characteristic of the peripheral nervous system. Proc Natl Acad Sci 89: 1183-1189. doi:10.1073/pnas.89.5.1983

Goedert M, Jakes R, Spillantini MG, Hasegawa M, Smith MJ, Crowther RA. 1996. Assembly of microtubule-associated protein tau into Alzheimer-like filaments induced by sulphated glycosaminoglycans. Nature 383: 550-553. doi:10.1038/ $383550 \mathrm{a} 0$

Goedert M, Eisenberg DS, Crowther RA. 2017. Propagation of tau aggregates and neurodegeneration. Annu Rev Neurosci 40: 189-210. doi:10.1146/annurev-neuro-072116-031153

Gustke N, Trinczek B, Biernat J, Mandelkow EM, Mandelkow E. 1994. Domains of tau protein and interactions with microtubules. Biochemistry 33: 9511-9522. doi:10.1021/bi0019 $8 \mathrm{a} 017$

Haj-Yahya M, Lashuel HA. 2018. Protein semisynthesis provides access to tau disease-associated post-translational modifications (PTMs) and paves the way to deciphering the tau PTM code in health and diseased states. J Am Chem Soc 140: 66116621. doi:10.1021/jacs.8b02668

Houlden H, Baker M, Morris HR, MacDonald N, PickeringBrown S, Adamson J, Lees AJ, Rossor MN, Quinn P, Kertesz A, et al. 2001. Corticobasal degeneration and progressive 
supranuclear palsy share a common tau haplotype. Neurology 56: 1702-1706. doi:10.1212/WNL.56.12.1702

Hutton M, Lendon CL, Rizzu P, Baker M, Froelich S, Houlden H, Pickering-Brown S, Chakraverty S, Isaacs A, Grover A, et al. 1998. Association of missense and 5 '-splice site mutations in tau with inherited dementia FTDP-17. Nature 393: 702-705. doi: $10.1038 / 31508$

Iqbal K, Liu F, Gong CX. 2016. Tau and neurodegenerative disease: the story so far. Nat Rev Neurol 12: 15-27. doi:10 .1038 /nrneurol.2015.225

Jackson SJ, Kerridge C, Cooper J, Cavallini A, Falcon B, Cella CV, Landi A, Szekeres PG, Murray TK, Ahmed Z, et al. 2016. Short fibrils constitute the major species of seedcompetent tau in the brains of mice transgenic for human P301S tau. J Neurosci 36: 762-772. doi:10.1523/JNEURO SCI.3542-15.2016

Janning D, Igaev M, Sündermann F, Brühmann J, Beutel O, Heinisch JJ, Bakota L, Piehler J, Junge W, Brandt R. 2014. Single-molecule tracking of tau reveals fast kiss-and-hop interaction with microtubules in living neurons. Mol Biol Cell 25: 3541-3551. doi:10.1091/mbc.e14-06-1099

Jicha GA, Bowser R, Kazam IG, Davies P. 1997. Alz-50 and MC1 , a new monoclonal antibody raised to paired helical filaments, recognize conformational epitopes on recombinant tau. J Neurosci Res 48: 128-132. doi:10.1002/(SICI)10974547(19970415)48:2<128::AID-JNR5>3.0.CO;2-E

Kellogg EH, Hejab NMA, Poepsel S, Downing KH, DiMaio F, Nogales E. 2018. Near-atomic model of microtubule-tau interactions. Science 360: 1242-1246. doi:10.1126/science.aat 1780

Koolen DA, Viussers LE, Pfundt R, de Leeuw N, Knight SJ, Regan R, Kooy RF, Reyniers E, Romano C, Fichera M, et al. 2006. A new chromosome 17q21.31 microdeletion syndrome associated with a common inversion polymorphism. Nat Genet 38: 999-1001. doi:10.1038/ng1853

Koolen DA, Kramer JM, Neveling K, Nillsen WM, Moore-Barton HL, Elmslie EV, Toutain A, Amiel J, Malan V, Tsai ACH, et al. 2012. Mutations in the chromatin modifier gene KANSL1 cause the $17 \mathrm{q} 21.31$ microdeletion syndrome. Nat Genet 44: 639-641. doi:10.1038/ng.2262

Lee G, Newman ST, Gard DL, Band H, Panchamoorthy G. 1998. Tau interacts with src-family non-receptor tyrosine kinases. $J$ Cell Sci 111: 3167-3177.

Min SW, Cho SH, Zhou Y, Schroeder S, Haroutunian V, Seeley WW, Huang EJ, Shen Y, Masliah E, Mukherjee C, et al. 2010. Acetylation of tau inhibits its degradation and contributes to tauopathy. Neuron 67: 953-966. doi:10.1016/j.neuron.2010 .08 .044

Moore CL, Huang MH, Robbenolt SA, Voss KR, Combs B, Gamblin TC, Goux WJ. 2011. Secondary nucleating sequences affect kinetics and thermodynamics of tau aggregation. Biochemistry 50: 10876-10886. doi:10.1021/bi2014745

Morris HR, Baker M, Yasojima K, Houlden H, Khan MN, Wood NW, Hardy J, Grossman M, Trojanowski JQ, Revesz T, et al. 2002. Analysis of tau haplotypes in Pick's disease. Neurology 59: 443-445. doi:10.1212/WNL.59.3.443

Niewidok B, Igaev M, Sündermann F, Janning DF, Bakota L, Brandt R. 2016. Presence of a carboxy-terminal pseudorepeat and disease-like pseudohyperphosphorylation critically influence tau's interaction with microtubules in axon-like processes. Mol Biol Cell 27: 3537-3549. doi:10.1091/mbc .e16-06-0402

Pastor P, Ezquerra M, Muñoz E, Martí MJ, Blesa R, Tolosa E, Oliva R. 2000. Significant association between the tau gene A0/A0 genotype and Parkinson's disease. Ann Neurol 47: 242-245. doi:10.1002/1531-8249(200002)47:2<242::AID-ANA $16>3.3 . \mathrm{CO} ; 2-\mathrm{C}$

Pérez M, Valpuesta JM, Medina M, Montejo de Garcini E, Avila J. 1996. Polymerization of tau into filaments in the presence of heparin: the minimal sequence required for tau-tau interaction. J Neurochem 67: 1183-1190. doi:10.1046/j.1471-4159.1996 .67031183.x

Poorkaj P, Bird TD, Wijsman E, Nemens E, Garruto RM, Anderson L, Andreadis A, Wiederholt WC, Raskind M, Schellen- berg GD. 1998. Tau is a candidate gene for chromosome 17 frontotemporal dementia. Ann Neurol 43: 815-825. doi: 10 $.1002 /$ ana.410430617

Qiang L, Sun X, Austin TO, Muralidharan H, Jean DC, Liu M, Yu W, Baas PW. 2018. Tau does not stabilize axonal microtubules but rather enables them to have long labile domains. Curr Biol 28: 2181-2189. doi:10.1016/j.cub.2018 .05 .045

Sharp AJ, Hansen S, Selzer RR, Cheng Z, Regan R, Hurst JA, Stewart H, Price SM, Blair E, Hennekam RC, et al. 2006. Discovery of previously unidentified genomic disorders from the duplication architecture of the human genome. Nat Genet 38: 1038-1042. doi:10.1038/ng1862

Shaw-Smith C, Pittman AM, Willatt L, Martin H, Rickman L, Gribble S, Curley R, Cumming S, Dunn C, Kalaitzopoulos D, et al. 2006. Microdeletion encompassing MAPT at chromosome $17 \mathrm{q} 21.31$ is associated with developmental delay and learning disability. Nat Genet 38: 1032-1037. doi:10.1038/ ng1858

Spillantini MG, Murrell JR, Goedert M, Farlow MR, Klug A, Ghetti B. 1998. Mutation in the tau gene in familial multiple system tauopathy with presenile dementia. Proc Natl Acad Sci 95: 7737-7741. doi:10.1073/pnas.95.13.7737

Stefanoska K, Volkerling A, Bertz J, Poljak A, Ke YD, Ittner LM. 2018. An N-terminal motif unique to primate tau enables differential protein-protein interactions. $J$ Biol Chem 293: 3710 3719. doi:10.1074/jbc.RA118.001784

Stefansson H, Helgason A, Thorleifsson G, Steinthorsdottir V, Masson G, Barnard J, Baker A, Jonasdottir A, Ingason A, Gudnadottir VG, et al. 2005. A common inversion under selection in Europeans. Nat Genet 37: 129-137. doi:10.1038/ ng1508

Tuerde D, Kimura T, Miyasaka T, Furusawa K, Shimozawa A, Hasegawa M, Ando K, Hisanaga SI. 2018. Isoform-independent and -dependent phosphorylation of microtubule-associated protein tau in mouse brain during postnatal development. J Biol Chem 293: 1781-1793. doi:10.1074/jbc .M117.798918

Von Bergen M, Friedhoff P, Biernat J, Heberle J, Mandelkow EM, Mandelkow E. 2000. Assembly of $\tau$ protein into Alzheimer paired helical filaments depends on a local sequence motif $\left({ }^{306}\right.$ VQIVYK $\left.^{311}\right)$ forming $\beta$ structure. Proc Natl Acad Sci 97: 5129-5134. doi:10.1073/pnas.97.10.5129

Wegmann S, Eftakharzadeh B, Tepper K, Zoltowaka KM, Bennett RE, Dujardin S, Laskowski PR, MacKenzie D, Kamath T, Commins C, et al. 2018. Tau protein liquid-liquid phase separation can initiate tau aggregation. EMBO J 37: e98049. doi:10.15252/embj.201798049

Wischik CM, Novak M, Thogersen HC, Edwards PC, Runswick MJ, Jakes R, Walker JE, Milstein C, Roth M, Klug A. 1988a. Isolation of a fragment of tau derived from the core of the paired helical filament of Alzheimer disease. Proc Natl Acad Sci 85: 4506-4510. doi:10.1073/pnas.85.12.4506

Wischik CM, Novak M, Edwards PC, Klug A, Tichelaar W, Crowther RA. 1988b. Structural characterization of the core of the paired helical filament of Alzheimer disease. Proc Natl Acad Sci 85: 4884-4888. doi:10.1073/pnas.85.13.4884

Zhang CC, Zhu JX, Wan Y, Tan L, Wang HF, Yu JT, Tan L. 2017a. Meta-analysis of the association between variants in MAPT and neurodegenerative diseases. Oncotarget 8: 4499445007. doi:10.18632/oncotarget.16690

Zhang X, Lin Y, Eschmann NA, Zhou H, Rauch JN, Hernandez I, Guzman E, Kosik KS, Han S. 2017b. RNA stores tau reversibly in complex coacervates. PLoS Biol 15: e2002183. doi:10 .1371/journal.pbio.2002183

Zhong Q, Congdon EE, Nagaraja HN, Kuret J. 2012. Tau isoform composition influences rate and extent of filament formation. $J$ Biol Chem 287: 20711-20719. doi:10.1074/jbc.M112.364067

Zollino M, Orteschi D, Murdolo M, Lattante S, Battaglia D, Stefanini C, Mercuri E, Chiurazzi P, Neri G, Marangi G. 2012. Mutations in KANSL1 cause the $17 \mathrm{q} 21.31$ microdeletion syndrome phenotype. Nat Genet 44: 636-638. doi:10.1038/ng .2257 


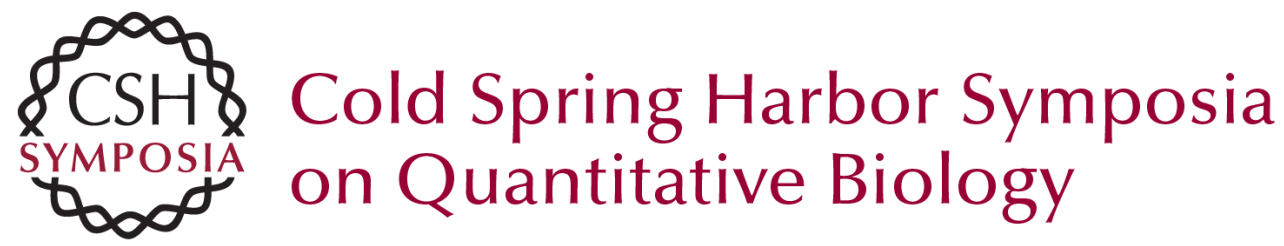

\section{Distinct Conformers of Assembled Tau in Alzheimer's and Pick's Diseases}

Michel Goedert, Benjamin Falcon, Wenjuan Zhang, et al.

Cold Spring Harb Symp Quant Biol 2018 83: 163-171 originally published online March 18, 2019 Access the most recent version at doi:10.1101/sqb.2018.83.037580

References This article cites 58 articles, 20 of which can be accessed free at: http://symposium.cshlp.org/content/83/163.full.html\#ref-list-1

Creative This article is distributed under the terms of the

Commons http://creativecommons.org/licenses/by-nc/4.0/, which permits reuse and License redistribution, except for commercial purposes, provided that the original author and source are credited.

Email Alerting Receive free email alerts when new articles cite this article - sign up in Service the box at the top right corner of the article or click here. 